\title{
Effective hydrogen diffusion coefficient for solidifying aluminium alloys
}

\author{
M. Felberbaum ${ }^{\text {a,**, E. Landry-Désy }}{ }^{\mathrm{a}}$, L. Weber ${ }^{\mathrm{b}}$, M. Rappaz ${ }^{\mathrm{a}}$ \\ a Laboratoire de Simulation des Matériaux, École Polytechnique Fédérale de Lausanne, EPFL-STI-IMX-LSMX, \\ Station 12, CH-1015 Lausanne, Switzerland \\ ${ }^{\mathrm{b}}$ Laboratoire de Métallurgie Mécanique, École Polytechnique Fédérale de Lausanne, EPFL-STI-IMX-LMM, Station 12, CH-1015 Lausanne, Switzerland
}

Received 31 August 2010; received in revised form 8 December 2010; accepted 10 December 2010

\begin{abstract}
An effective hydrogen diffusion coefficient has been calculated for two solidifying $\mathrm{Al}-4.5 \mathrm{wt} . \% \mathrm{Cu}$ and $\mathrm{Al}-10 \mathrm{wt} . \% \mathrm{Cu}$ alloys as a function of the volume fraction of solid. For this purpose, in situ X-ray tomography was performed on these alloys. For each volume fraction of solid between 0.6 and 0.9 , a representative volume element of the microstructure was extracted. Solid and liquid voxels were assimilated to solid and liquid nodes in order to solve the hydrogen diffusion equation based on the chemical potential and using a finite volume formulation. An effective hydrogen diffusion coefficient based on the volume fraction of solid only could be deduced from the results of the numerical model at steady state. The results are compared with various effective medium theories.
\end{abstract}

(c) 2010 Acta Materialia Inc. Published by Elsevier Ltd. All rights reserved.

Keywords: Solidification; Aluminium alloys; Simulation; Synchrotron radiation; Hydrogen diffusion

\section{Introduction}

The detrimental effects of hydrogen on the mechanical properties of cast aluminium alloys have been studied for many years [1]. Hydrogen is the only gas that is significantly soluble in liquid aluminium; moreover, it is strongly segregated during solidification since the solid solubility is substantially lower [2]. Concomitant to the partitioning of hydrogen during solidification, the maximum solubility decreases as a result of both the temperature decrease and the pressure drop across the mushy zone induced by solidification shrinkage. Once the gas concentration in the liquid exceeds the solubility, pores can nucleate and grow [3].

Once a pore has nucleated, it grows and hence dissolved hydrogen must diffuse through the solidifying alloy towards the pore. Since hydrogen diffuses very rapidly (the hydrogen diffusion coefficient in liquid aluminium $D_{\ell} \approx 10^{-7} \mathrm{~m}^{2} \mathrm{~s}^{-1}$ at the melting point [2]), most porosity

\footnotetext{
* Corresponding author.

E-mail address: milan.felberbaum@a3.epfl.ch (M. Felberbaum).
}

models assume a lever rule for hydrogen mass conservation and neglect diffusion [4-6]. However, it has been shown that pore growth can be limited by hydrogen diffusion $[7,8]$. In order to take the influence of hydrogen diffusion into account, previous models assumed that the pore is "fed" through the liquid-gas interface only $[8,9]$. For pores growing in the mushy zone, where both solid and liquid are in contact with the pore, a pore-liquid interfacial area "fraction" (a function of the volume fraction of solid) must then be estimated, which is a rather difficult task. Moreover, diffusion of hydrogen through the solid can no longer be neglected when the solid fraction is large.

For these reasons, Atwood et al. [10] introduced an effective hydrogen diffusion coefficient $\langle D\rangle$ as a function of the local solid fraction. These authors used an effective medium theory initially developed for the transverse thermal conductivity of a unidirectional fibre composite [11], i.e. they directly replaced the thermal conductivity of the phases by their diffusion coefficient in Markworth's equation for the case of perfect transfer at the interface. However, this equation applies to a two-dimensional (2D) structure and is valid only at low solid fractions 
and for a specific morphology. A freezing aluminium alloy exhibits a much more complex two-phase 3D structure, which furthermore undergoes a topological change near $g_{s}=0.9$ : from isolated solid grains (or dendrites) surrounded by the liquid, the microstructure transforms into a coalesced solid with isolated liquid pockets $[12,13]$. Moreover, replacing the phase thermal conductivity directly by the diffusion coefficient is inappropriate since it is the chemical potential and not the composition that is the equivalent of the temperature field. Analytical calculation of an effective diffusion coefficient in a two-phase material should therefore be made based on chemical potential gradients and atomic mobility rather than composition gradients and diffusion coefficients.

In the present study, an effective hydrogen diffusion coefficient is calculated for a representative volume element (RVE) of a solidifying aluminium alloy. The RVE is extracted from in situ X-ray tomography measurements performed at the European Radiation Synchrotron Facility (ESRF) in Grenoble (France) on $\mathrm{Al}-4.5 \mathrm{wt} . \% \mathrm{Cu}$ and $\mathrm{Al}-$ 10 wt. \% Cu samples. Each of the reconstructed 3D images recorded at various temperatures provides a $3 \mathrm{D}$ mesh for a finite volume method (FVM) calculation of diffusion based on the chemical potential.

Using a time-explicit scheme, the average hydrogen flux across the RVE and thus an effective diffusion coefficient are computed and compared with analytical solutions based on effective medium theories.

\section{Experimental}

First, aluminium-copper samples were solidified in an induction furnace under argon atmosphere. From pure aluminium (99.995\% purity) and oxygen-free high-conductivity copper shots, $\mathrm{Al}-4.5 \mathrm{wt} . \% \mathrm{Cu}$ and $\mathrm{Al}-10 \mathrm{wt} . \% \mathrm{Cu}$ samples were produced. Specimens of about $4.5 \mathrm{~mm}^{3}$ (1.4 $\mathrm{mm}$ diameter, $3 \mathrm{~mm}$ high) were then extracted from these samples and analyzed using X-ray tomography. These tomography measurements are similar to those of Ref. [14] and will not be detailed here. In brief, the rotating sample was heated in a fixed resistance furnace up to its melting temperature. It was then cooled down at $3 \mathrm{~K} \mathrm{~min}^{-1}$ and a scan was taken every $1.28 \mathrm{~min}$ to characterize the microstructural evolution during solidification. The camera pixel size was set to $h=2.8 \mu \mathrm{m} \mathrm{pixel}^{-1}$ and 500 radiography projections, each made of $512 \times 512$ pixels, were recorded in $51 \mathrm{~s}$ during a $180^{\circ}$ rotation. After reconstruction, the images were cropped to a size of $200 \times$ $200 \times 200$ pixels, i.e. $560 \times 560 \times 560 \mu^{3}$, in order to perform $3 \mathrm{D}$ calculations with a personal computer. Depending on its grey level, a phase index $\psi=1$ or 0 for solid or liquid, respectively, was attributed to each voxel. The $3 \mathrm{D}$ volume information then served as the mesh for the timeexplicit finite volume model, with a phase index (solid/ liquid) at each mesh point obtained from the tomography binary voxel index.

\section{Theory}

\subsection{Diffusion}

In the diffusion process, the flux of hydrogen atoms $\mathrm{H}$, $\mathscr{J}^{\mathrm{H}}$, at any point in the lattice is proportional to the chemical potential gradient $\nabla \mu^{\mathrm{H}}$ and is given by [15]:

$\mathscr{J}^{\mathrm{H}}=-M^{\mathrm{H}} C^{\mathrm{H}} \nabla \mu^{\mathrm{H}}$

where $M^{\mathrm{H}}$ is the mobility coefficient of $\mathrm{H}$ atoms, $C^{\mathrm{H}}$ is the local molar concentration (in $\mathrm{mol} \mathrm{m}^{-3}$ ) and the flux is given in $\mathrm{mol} \mathrm{m}^{-2} \mathrm{~s}^{-1}$. Combining Eq. (1) with the GibbsDuhem relationship, and introducing the molar volume $V^{m}=X^{\mathrm{H}} / C^{\mathrm{H}}$, where $X^{\mathrm{H}}$ is the molar composition of $\mathrm{H}$, the following equation is obtained [15]:

$\mathscr{J}^{\mathrm{H}}=-M^{\mathrm{H}} \frac{R T}{V^{m}}\left(1+\frac{\mathrm{d} \ln f^{\mathrm{H}}}{\mathrm{d} \ln X^{\mathrm{H}}}\right) \nabla X^{\mathrm{H}}$

where $f^{\mathrm{H}}$ is the activity coefficient of hydrogen, i.e. $f^{\mathrm{H}}=a^{\mathrm{H}} /$ $X^{\mathrm{H}}$, with $a^{\mathrm{H}}$ the corresponding chemical activity. A comparison with Fick's first law allows the diffusion coefficient $D^{\mathrm{H}}\left(\mathrm{m}^{2} \mathrm{~s}^{-1}\right)$ to be related to the atomic mobility $M^{\mathrm{H}}$ :

$D^{\mathrm{H}}=M^{\mathrm{H}} R T\left(1+\frac{\mathrm{d} \ln f^{\mathrm{H}}}{\mathrm{d} \ln X^{\mathrm{H}}}\right)$

For dilute solutions such as the Al-H system, where $X^{\mathrm{H}} \rightarrow$ 0 and $f^{\mathrm{H}}=a^{\mathrm{H}} / X^{\mathrm{H}} \approx$ constant (Henry's law), the term in parentheses is equal to 1 . The diffusion coefficient is thus linked simply to the atomic mobility $M^{\mathrm{H}}$ via the following relationship:

$D^{\mathrm{H}}=M^{\mathrm{H}} R T$

In order to solve the diffusion equation for hydrogen in a heterogeneous material, i.e. one made of solid and liquid, it is necessary to use a variable that is continuous at interfaces. Assuming local equilibrium at interfaces, one has $\mu_{\ell}^{\mathrm{H}}=\mu_{s}^{\mathrm{H}}$ and thus it is appropriate to calculate diffusion in any part of the RVE from the equation:

$\mathbf{J}^{\mathrm{H}}=-M^{\mathrm{H}} X^{\mathrm{H}} \nabla \mu^{\mathrm{H}}$

This equation is similar to Eq. (1), but both parts of the equation have been divided by the molar volume, so that the flux $\mathbf{J}^{\mathrm{H}}$ is now given in $\mathrm{m} \mathrm{s}^{-1}$. The conservation of hydrogen is given by Fick's second law, which becomes:

$\frac{\partial X^{\mathrm{H}}}{\partial t}=-\boldsymbol{\nabla} \cdot \mathbf{J}^{\mathrm{H}}=\boldsymbol{\nabla} \cdot\left(M^{\mathrm{H}} X^{\mathrm{H}} \boldsymbol{\nabla} \mu^{\mathrm{H}}\right)$

This equation will be used and further developed in Section 4.1 to model hydrogen diffusion through a mushy zone microstructure.

\subsection{Chemical potentials}

In order to use Eq. (6), the chemical potential of hydrogen dissolved in the liquid and in the solid must be known. Qiu et al. [16] performed a thermodynamic evaluation of the $\mathrm{Al}-\mathrm{H}$ phase diagram. They gave a direct relationship 
between the chemical potential and the hydrogen molar fraction. The liquid phase was modeled as a substitutional solution between $\mathrm{Al}$ and $\mathrm{H}$, with its molar energy given by a regular solution at a fixed pressure:

$$
\begin{aligned}
G_{\ell}^{m}={ }^{\circ} G_{\ell}^{\mathrm{Al}} X^{\mathrm{Al}}+{ }^{\circ} G_{\ell}^{\mathrm{H}} X^{\mathrm{H}}+R T\left(X^{\mathrm{Al}} \ln X^{\mathrm{Al}}+X^{\mathrm{H}} \ln X^{\mathrm{H}}\right) \\
+\Omega_{\ell} X^{\mathrm{Al}} X^{\mathrm{H}}
\end{aligned}
$$

where $X^{\mathrm{Al}}$ and $X^{\mathrm{H}}$ are the mole fractions of $\mathrm{Al}$ and $\mathrm{H}$, respectively, ${ }^{\circ} G_{\ell}^{\mathrm{Al}}$ and ${ }^{\circ} G_{\ell}^{\mathrm{H}}$ are their corresponding Gibbs free energy in the liquid state, and $\Omega_{\ell}$ is the interaction parameter. The chemical potential of hydrogen in the liquid, $\mu_{\ell}^{\mathrm{H}}$, is thus given by:

$\mu_{\ell}^{\mathrm{H}}={ }^{\circ} G_{\ell}^{\mathrm{H}}+\Omega_{\ell}\left(1-X^{\mathrm{H}}\right)^{2}+R T \ln X^{\mathrm{H}}$

Unfortunately, the same procedure cannot be applied to the solid phase, because ${ }^{\circ} G_{s}^{\mathrm{H}}$ is unknown. However, the Gibbs free energy curve of the solid $G_{s}^{m}$ is known from Ref. [16] and the chemical potential at any composition can be found from the tangent rule, i.e. from:

$\frac{\partial G_{s}^{m}}{\partial X}=\frac{\mu_{s}^{\mathrm{H}}-G_{s}^{m}\left(X^{\mathrm{H}}\right)}{1-X^{\mathrm{H}}} \Rightarrow \mu_{s}^{\mathrm{H}}=\frac{\partial G_{s}^{m}}{\partial X^{\mathrm{H}}}\left(1-X^{\mathrm{H}}\right)+G_{s}^{m}\left(X^{\mathrm{H}}\right)$

Eqs. (8) and (9) provide both $\mu_{\ell}^{\mathrm{H}}$ and $\mu_{s}^{\mathrm{H}}$ as a function of $X^{\mathrm{H}}$, which allows the calculation of the chemical potential gradient appearing in Eq. (6) for both phases.

\section{Model}

\subsection{Discretization}

An explicit time-stepping FVM scheme was used to solve Eq. (6) for an RVE containing solid and liquid voxels. Integrating this equation over the volume $V_{i}=h^{3}$ of a mesh (voxel) point $i$, where $h$ is the mesh (voxel) size, and using the divergence theorem, one has:

$h^{3} \frac{\Delta X_{i}^{\mathrm{H}}}{\Delta t}=-h^{2} \sum_{j} \mathbf{J}_{i j}^{\mathrm{H}} \cdot \mathbf{n}_{j}=h^{2} \sum_{j}\left(M^{\mathrm{H}} X^{\mathrm{H}}\right)_{i j} \nabla \mu_{i j}^{\mathrm{H}} \cdot \mathbf{n}_{j}$

where $\mathbf{n}_{j}$ is the outward-pointing unit normal of the surface element separating node $i$ and its neighbour $j, \mathbf{J}_{i j}^{\mathrm{H}}$ is the hydrogen flux exchanged between these nodes, and $\left(M^{\mathrm{H}} X^{\mathrm{H}}\right)_{i j}$ is a combination of mobilities and molar compositions of hydrogen at both nodes that needs to be averaged properly. If the neighbour $j$ of node $i$ has the same phase index (i.e. both nodes are solid or both are liquid), the problem is trivial and $\left(M^{\mathrm{H}} X^{\mathrm{H}}\right)_{i j}=M_{i}^{\mathrm{H}} X_{i}^{\mathrm{H}}$. The flux exchange between the nodes in this case is simply:

$\mathbf{J}_{i j}^{\mathrm{H}} \cdot \mathbf{n}_{j} \cong M_{i}^{\mathrm{H}} X_{i}^{\mathrm{H}} \frac{\mu_{j}^{\mathrm{H}}-\mu_{i}^{\mathrm{H}}}{\mathbf{r}_{j}-\mathbf{r}_{i}} \cdot \mathbf{n}_{j}=M_{i}^{\mathrm{H}} X_{i}^{\mathrm{H}} \frac{\mu_{j}^{\mathrm{H}}-\mu_{i}^{\mathrm{H}}}{h}$

The positions of nodes $i$ and $j$ are given by $\mathbf{r}_{i}$ and $\mathbf{r}_{j}$, respectively, and the summation is made over the six nearestneighbours nodes $j$ at the front/back, right/left and top/ bottom of node $i$.
If the two nodes are not of the same phase, care must be taken in calculating $\left(M^{\mathrm{H}} X^{\mathrm{H}}\right)_{i j}$. Since the chemical potential is continuous across the interface between two nodes, we call $\mu_{i j}^{\mathrm{H} *}$ the chemical potential at half distance between nodes $i$ and $j$. The hydrogen flux on both sides of the interface being equal, one has:

$M_{j}^{\mathrm{H}} X_{j}^{\mathrm{H}} \frac{\mu_{j}^{\mathrm{H}}-\mu_{i j}^{\mathrm{H} *}}{h / 2}=M_{i}^{\mathrm{H}} X_{i}^{\mathrm{H}} \frac{\mu_{i j}^{\mathrm{H} *}-\mu_{i}^{\mathrm{H}}}{h / 2}$

or

$\mu_{i j}^{\mathrm{H} *}=\frac{\left(M^{\mathrm{H}} X^{\mathrm{H}} \mu^{\mathrm{H}}\right)_{i}+\left(M^{\mathrm{H}} X^{\mathrm{H}} \mu^{\mathrm{H}}\right)_{j}}{\left(M^{\mathrm{H}} X^{\mathrm{H}}\right)_{i}+\left(M^{\mathrm{H}} X^{\mathrm{H}}\right)_{j}}$

Therefore the flux is given in this case by:

$\mathbf{J}_{i j}^{\mathrm{H}} \cdot \mathbf{n}_{j}=\left(M^{\mathrm{H}} X^{\mathrm{H}}\right)_{i j} \frac{\mu_{j}^{\mathrm{H}}-\mu_{i}^{\mathrm{H}}}{h}$

where $\left(M^{\mathrm{H}} X^{\mathrm{H}}\right)_{i j}$ is given by:

$\left(M^{\mathrm{H}} X^{\mathrm{H}}\right)_{i j}=2 \frac{\left(M^{\mathrm{H}} X^{\mathrm{H}}\right)_{i}\left(M^{\mathrm{H}} X^{\mathrm{H}}\right)_{j}}{\left(M^{\mathrm{H}} X^{\mathrm{H}}\right)_{i}+\left(M^{\mathrm{H}} X^{\mathrm{H}}\right)_{j}}$

Without too much of a surprise, we retrieve an average (mobility-composition) made of two half-elements of thickness $h / 2$ put in series for calculating the flux between the two nodes. Note that this equation retrieves Eq. (11) when the two considered nodes belong to the same phase, i.e. $M_{i}^{\mathrm{H}}=M_{j}^{\mathrm{H}}$. Strictly speaking, in this case one should still average the compositions of the nodes $i$ and $j$ as in Eq. (15) and not take just $X_{i}^{\mathrm{H}}$ as in Eq. (11). However, being in the same phase for both nodes, $X_{i}^{\mathrm{H}} \cong X_{j}^{\mathrm{H}}$ and thus both formulations of the fluxes are almost equivalent.

Having expressed the hydrogen flux $\mathbf{J}_{i j}^{\mathrm{H}} \cdot \mathbf{n}_{j}$ between any two nodes $i$ and $j$, the time-explicit scheme is equivalent to calculating at the new time step $(t+\Delta t)$ the compositions $X_{i}^{\mathrm{H}}(t+\Delta t)$ at all the nodes from the previous compositions $X_{i}^{\mathrm{H}}(t)$ and the known chemical potentials $\mu_{i}^{\mathrm{H}}(t)=$ $\mu^{\mathrm{H}}\left(X_{i}^{\mathrm{H}}(t)\right)$, i.e.:

$$
\begin{aligned}
X_{i}^{\mathrm{H}}(t+\Delta t)= & X_{i}^{\mathrm{H}}(t)+\frac{\Delta t}{h} \sum_{j}\left(M^{\mathrm{H}} X^{\mathrm{H}}\right)_{i j}(t) \\
& \times \frac{\mu_{j}^{\mathrm{H}}(t)-\mu_{i}^{\mathrm{H}}(t)}{h}
\end{aligned}
$$

The algorithm is simple and can be summarized as follows:

1. calculate the mean mobility-composition $\left(M^{\mathrm{H}} X^{\mathrm{H}}\right)_{i j}(t)$ at all interfaces between nodes $i$ and $j$, using Eq. (15), the phase index of the nodes, the mobility of hydrogen in the solid and liquid, and the compositions $X_{i}^{\mathrm{H}}(t)$ at all nodes at time $t$,

2. calculate the chemical potential $\mu_{i}^{\mathrm{H}}(t)$ at each node using Eqs. (8) and (9),

3. calculate the hydrogen fluxes exchanged between the nodes, $\mathbf{J}_{i j}^{\mathrm{H}} \cdot \mathbf{n}_{j}$, using Eq. (14),

4. calculate the new composition $X_{i}^{\mathrm{H}}(t+\Delta t)$ at each node using Eq. (16), 
5. restart at step 1 until the end of the time steps (typically when steady state is reached).

We used a time-explicit discretization for two reasons. First, it avoids having to invert a sparse $\left(8 \times 10^{6}\right) \times$ $\left(8 \times 10^{6}\right)$ matrix. Second, the problem is non-linear, since the compositions also appear on the right-hand side of Eq. (10) and furthermore the relationships between chemical potential and composition are non-linear. If an implicit time-stepping scheme were to be used, inverse relations $X^{\mathrm{H}}\left(\mu^{\mathrm{H}}\right)$ would need to be derived. The simplicity of the explicit scheme is of course counterbalanced by the necessity of using small time steps. For a 3D linear problem of diffusion, it is recalled that the time step $\Delta t$ must satisfy the Fourier condition: $D^{\mathrm{H}} \Delta t / h^{2}<1 / 6$. This criterion is even more severe in our non-linear situation (see Table 1).

Concerning the boundary conditions applied at the frontiers of the $(560 \mu \mathrm{m})^{3} \mathrm{RVE}$, a zero flux condition is applied on the front, back, top and bottom frontiers of the volume. Dirichlet boundary conditions are then considered for the left and right frontiers (see Fig. 1):

- $\mu_{s}^{W}=\mu_{\ell}^{W}=\mu_{e q}^{\mathrm{H}}$ on the left (West) boundary, where $\mu_{e q}^{\mathrm{H}}$ corresponds to the equilibrium chemical potential of hydrogen at $933.6 \mathrm{~K}$ and $1 \mathrm{~atm}$,

- $\mu_{s}^{E}=\mu_{\ell}^{E}=-71856 \mathrm{~J} \mathrm{~mol}^{-1}$ on the right (East) boundary.

The chemical potential at the right frontier of the domain corresponds to a very low composition $\varepsilon=10^{-10}$ $(-)$ in the solid and $\varepsilon=10^{-10} / k_{0}^{\mathrm{H}}(-)$ in the liquid, where $k_{0}^{\mathrm{H}}$ is the partition coefficient of hydrogen in aluminium. Finally, the initial compositions $X_{i}^{\mathrm{H}}(t=0)$ are fixed to $\varepsilon=10^{-10}(-)$ everywhere in the domain except at the left frontier, where they are set to $X_{i, \ell}^{\mathrm{H}}\left(\mu_{e q}^{\mathrm{H}}\right)$ and $X_{i, s}^{\mathrm{H}}\left(\mu_{e q}^{\mathrm{H}}\right)$ at the liquid and solid nodes, respectively.

Finally, even if REVs are for different solid fractions, i.e. different temperatures for $\mathrm{Al}-\mathrm{Cu}$ alloys, the temperature and the pressure of the system are assumed to remain fixed at $933.6 \mathrm{~K}$ and $1 \mathrm{~atm}$, respectively. The influences of copper and of curvature on the Al-H equilibrium condition (Section 3.2) are also neglected. Thus, only the influence of the microstructure on diffusion is taken into account.

Table 1

Inputs values for the model (for $p=1 \mathrm{~atm}$ and $T=933.6 \mathrm{~K}$ ).

\begin{tabular}{lll}
\hline Entity & Value & Reference \\
\hline$\Delta t$ & $2.0 \mathrm{e}-6 \mathrm{~s}$ & - \\
Number of steps & $3 \mathrm{e} 6$ & - \\
$D_{s}^{\mathrm{H}}$ & $4.48 \mathrm{e}-8 \mathrm{~m}^{2} \mathrm{~s}^{-1}$ & {$[2]$} \\
$D_{\ell}^{\mathrm{H}}$ & $1.09 \mathrm{e}-7 \mathrm{~m} \mathrm{~s}^{2}$ & {$[2]$} \\
$X_{\ell, e q}^{\mathrm{H}}$ & $1.56037 \mathrm{e}-5$ & {$[16]$} \\
$X_{s, e q}^{\mathrm{H}}$ & $9.02644 \mathrm{e}-7$ & {$[16]$} \\
$\mu_{e q}^{\mathrm{H}}$ & $-1099.54 \mathrm{~J} \mathrm{~mol}^{-1}$ & {$[16]$} \\
${ }^{\circ} G_{\ell}^{\mathrm{H}}$ & $44144.5 \mathrm{~J} \mathrm{~mol}^{-1}$ & {$[16]$} \\
$\Omega_{s}$ & $6877.67 \mathrm{~J} \mathrm{~mol}^{-1}$ & {$[16]$} \\
$\Omega_{\ell}$ & $40624.3 \mathrm{~J} \mathrm{~mol}^{-1}$ & {$[16]$} \\
\hline
\end{tabular}

\subsection{Diffusion coefficient averaging}

Dealing with the diffusion of hydrogen only, we omit for the sake of simplicity the superscript " $\mathrm{H}$ " in the following developments and keep only the phase index $v(v=s$ or $\ell)$ where necessary. Starting with Eqs. (4) and (5) and using the definition of the chemical activity $a_{v}$ of hydrogen in a given phase $v$, i.e. $a_{v}=\exp \left(-\left({ }^{\circ} G_{v}-\mu_{v}\right) / R T\right)$, or alternatively starting from $a_{v}=f_{v} X_{v}$, it is easy to show that in each phase one has separately:

$\mathbf{J}_{v}=-D_{v} \nabla X_{v}=-D_{v} \nabla\left(\frac{a_{v}}{f_{v}}\right) \stackrel{\text { Henry }}{\cong}-\frac{D_{v}}{f_{v}} \nabla a_{v}=-\mathcal{D}_{v} \nabla a_{v}$

Note that one has assumed Henry's law again for taking the chemical activity coefficient of hydrogen $f_{v}$ out of the gradient. We have introduced a new entity $\mathfrak{D}_{v}=\frac{D_{v}}{f_{v}}$ that we call "mobility coefficient" of phase $v$ that in this case represents the ability of the phase to transport hydrogen. We see that not only the diffusion coefficient, but also the activity coefficient must be taken into account if one wants to derive effective properties for diffusion in a multi-phase material.

Now that we have a linear relationship between the flux $\mathbf{J}_{v}$ and the gradient of a quantity that is continuous across the interface (the activity $a$ ), we can use common models for establishing an effective mobility coefficient $\langle\mathfrak{D}\rangle$ and an average hydrogen flux $\langle\mathbf{J}\rangle$ in the two-phase RVE. From this average flux, we can then deduce an average diffusion coefficient $\langle D\rangle$, by considering an average composition $\langle X\rangle=g_{s} X_{s}+g_{\ell} X_{\ell}$, where $g_{s}$ and $g_{\ell}$ are the phase fractions. (Note that they can be mass or volume fractions, since we assume the same density for the solid and liquid phases.) In other words, we have:

$\langle\mathbf{J}\rangle=-\langle\mathfrak{D}\rangle \nabla a=-\langle D\rangle \boldsymbol{\nabla}\langle X\rangle$

Note that we have arbitrarily defined $\langle D\rangle$ such that $\langle D\rangle=\langle\mathbf{J}\rangle / \nabla\langle X\rangle$, where both $\langle\mathbf{J}\rangle$ and $\langle X\rangle$ are deduced from the finite volume computations.

Since:

$\langle X\rangle=X_{s} g_{s}+X_{\ell} g_{\ell}=\frac{a_{s}}{f_{s}} g_{s}+\frac{a_{\ell}}{f_{\ell}} g_{\ell}=a\left(\frac{g_{s}}{f_{s}}+\frac{g_{\ell}}{f_{\ell}}\right)$

one also has:

$\nabla\langle X\rangle=\left(\frac{g_{s}}{f_{s}}+\frac{g_{\ell}}{f_{\ell}}\right) \nabla a$

for an RVE in which the phase fractions are constant, and with $f_{s}$ and $f_{\ell}$ also being constant.

We finally have:

$\langle D\rangle=\langle\mathfrak{D}\rangle \cdot \frac{\nabla a}{\nabla\langle X\rangle}=\langle\mathfrak{D}\rangle\left(\frac{g_{s}}{f_{s}}+\frac{g_{\ell}}{f_{\ell}}\right)^{-1}$

The average diffusion coefficients obtained from the simulation results will be compared with analytical values deduced from the effective medium theories, which are briefly summarized in the next section. 


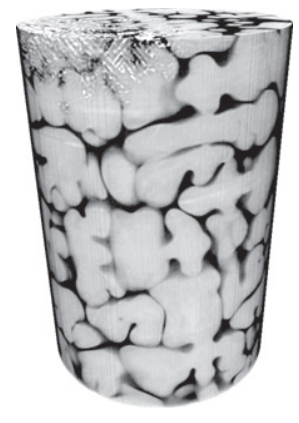

$\overline{500 \mu \mathrm{m}}$

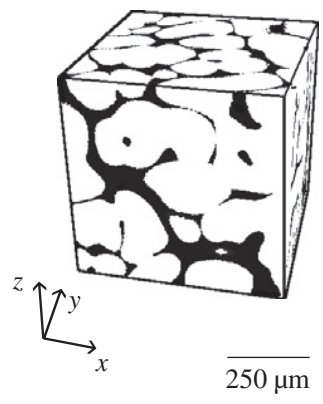

(b)

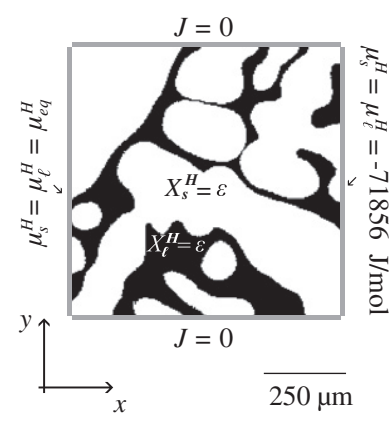

(c)

Fig. 1. (a) 3D volume rendering of a reconstructed solidifying $\mathrm{Al}-10 \mathrm{wt} . \% \mathrm{Cu}$ sample $\left(g_{s}=0.8\right)$. (b) Representative volume element. Solid regions are white $(\psi=1)$, whereas black represents liquid regions $(\psi=0)$. (c) $2 \mathrm{D}$ cross-section with the applied boundary conditions.

\subsection{Effective medium approximations}

Effective medium theories rely on the fact that there is a linear relationship between an average generalized flux, in our case $\langle\mathbf{J}\rangle$, and an average (or applied) generalized intensity, in our case $\nabla a$, continuous at the interface between various phases [17]. There are several ways to average the fluxes and thus properties such as $\langle\mathfrak{D}\rangle$. For a composite made of spherical particles (phase 2) in a matrix (phase 1), we consider the three most important ones:

- The self-consistent approximation (SC) [18]:

$$
\begin{aligned}
\langle\mathfrak{D}\rangle_{S C}= & \frac{1}{4}\left(\mathfrak{D}_{1}\left(3 g_{1}-1\right)+\mathfrak{D}_{2}\left(3 g_{2}-1\right)\right. \\
& \left.+\sqrt{\left(\mathfrak{D}_{1}\left(3 g_{1}-1\right)+\mathfrak{D}_{2}\left(3 g_{2}-1\right)\right)^{2}+8 \mathfrak{D}_{1} \mathfrak{D}_{2}}\right)
\end{aligned}
$$

- The Maxwell-type approximation [19], coinciding with the Hashin-Shtrikman (HS) upper bond:

$$
\langle\mathfrak{D}\rangle_{H S}=\mathfrak{D}_{1} \frac{2 \mathfrak{D}_{1} g_{1}+\mathfrak{D}_{2}\left(1+2 g_{2}\right)}{\mathfrak{D}_{1}\left(3-g_{1}\right)+\mathfrak{D}_{2}\left(1-g_{2}\right)}
$$

- The differential effective medium approximation (DEM) [20]:

$$
\frac{\mathfrak{D}_{2}-\langle\mathfrak{D}\rangle_{D E M}}{\mathfrak{D}_{2}-\mathfrak{D}_{1}}\left(\frac{\mathfrak{D}_{1}}{\langle\mathfrak{D}\rangle_{D E M}}\right)^{1 / 3}=1-g_{2}
$$

The SC approximation is implicit: it requires solving implicit equations for the effective properties and it treats each phase symmetrically: both phases are considered as inclusions in a composite matrix. Hashin and Shtrikman [21], on the other hand, derived explicit relationships for the best possible bounds on the effective conductivity of two-phase isotropic $3 \mathrm{D}$ composites given just the volume fraction information [17]. Finally the DEM implicit approximation scheme lies between the HS lower and upper bounds and is supposed to be a better approximation in the case of a two-phase material with high phase contrast $\mathfrak{D}_{2} / \mathfrak{D}_{1}$ (typically more than 4 [22]).

\section{Results and discussion}

Before calculations were performed on real microstructures obtained by X-ray tomography, validations using a fictitious arrangement of solid and liquid layers connected in parallel or series were performed (see Ref. [23] for details). Then computations were run on two different alloys ( $\mathrm{Al}-4.5 \mathrm{wt} . \% \mathrm{Cu}$ and $\mathrm{Al}-10 \mathrm{wt} . \% \mathrm{Cu}$ ) for four different volume fractions $\left(g_{s}=0.6 \rightarrow 0.9\right)$. About 10 days were needed for each computation on an Intel Xeon CPU@ $2.5 \mathrm{GHz}$ to reach steady state in an RVE made of $200 \times 200 \times 200$ volume elements. Fig. 2 shows the results (composition map on a cross-section in the $x y$ plane for each $g_{s}$ ) of 3D calculations after reach steady state has been reached. The grey level is proportional to the local hydrogen composition, with a logarithmic scale shown on the right. Liquid regions are the brightest and have only very small composition gradients (almost invisible on these figures because of the logarithmic scale) because of the highest solubility and diffusion coefficient. The iso-compositions in the solid are clearly visible and are not perpendicular to the $x$-axis (left-right direction), because of the influence of the tortuous 3D microstructure. Note that at $x=0$ the compositions in the solid and in the liquid are unequal, since the same equilibrium chemical potential (and not composition) has been imposed for both phases.

The calculated composition and flux of hydrogen were then averaged on each plane $x=$ constant to deduce $\langle X\rangle(x)$ (Eq. 19) and $\langle J\rangle$, respectively. When reach steady state is reached, the results of the computations show that in each plane $x=$ constant we have $\left\langle J_{x}\right\rangle \gg\left\langle J_{y}\right\rangle$ and $\left\langle J_{x}\right\rangle \gg\left\langle J_{z}\right\rangle$. Accordingly, we approximate $\langle J\rangle \approx\left\langle J_{x}\right\rangle$ (the component of $\langle J\rangle$ in the $x$ direction) to calculate the average hydrogen flux. Fig. 3 shows the average composition $\langle X\rangle(x)$ as a function of the distance from the plane $x=0$ for each solid fraction $g_{s}$ of an $\mathrm{Al}-4.5 \mathrm{wt} . \% \mathrm{Cu}$ alloy (note that a similar trend is obtained for the $\mathrm{Al}-10 \mathrm{wt} . \% \mathrm{Cu}$ alloy). All the 


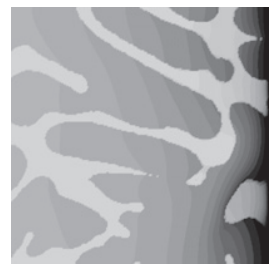

$g_{s}=0.6$

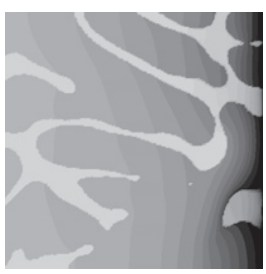

$g_{s}=0.7$

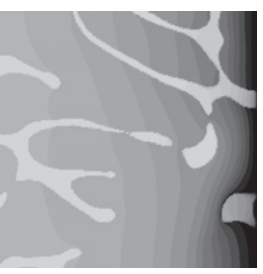

$g_{s}=0.8$

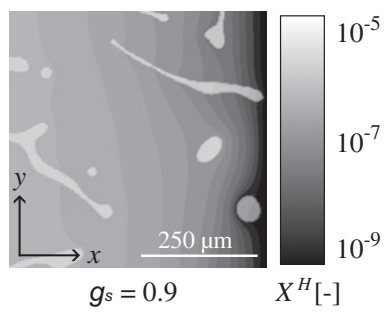

Fig. 2. Cross-sections in the $x y$-plane of the steady-state hydrogen composition maps for four solid fractions $g_{s}$. The logarithmic grey scales for the composition and the coordinate system are indicated on the right.

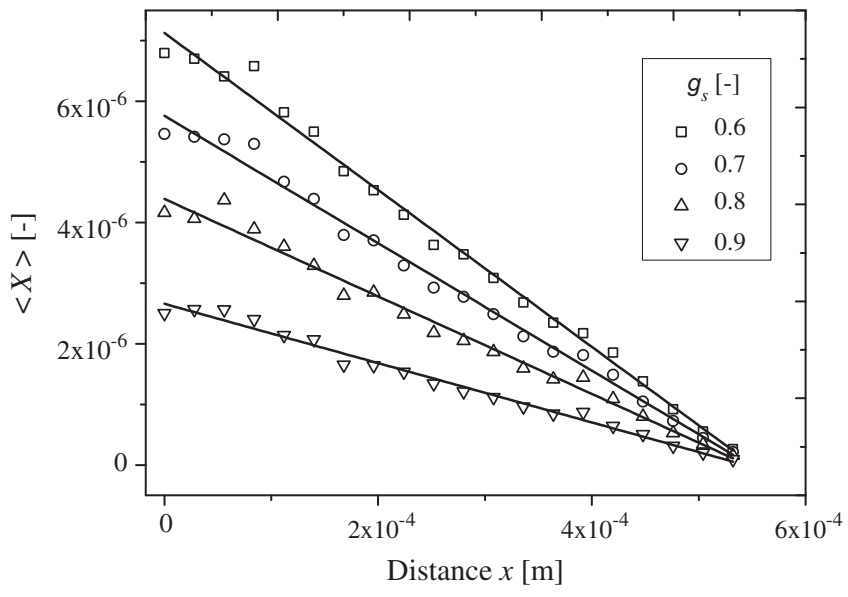

Fig. 3. Average hydrogen molar fraction profile along the $x$-axis for various volume fractions of solid in an $\mathrm{Al}-4.5 \mathrm{wt} . \% \mathrm{Cu}$ alloy. Each black line shows a linear fit with a correlation coefficient of 0.99 .

composition profiles appear to be linear, thus allowing a linear regression to be performed for all the volume fractions of solid. The points do not lie exactly on a single line, probably because of the tortuous shape of the mushy zone. Also, the solid fraction in each plane $x=$ constant differs slightly from the overall volumetric fraction of the RVE, thus skewing the results. Nevertheless, a correlation coefficient $R^{2} \geqslant 0.99$ is always obtained, which allows us to divide the flux by the average composition gradient $\nabla\langle X\rangle$

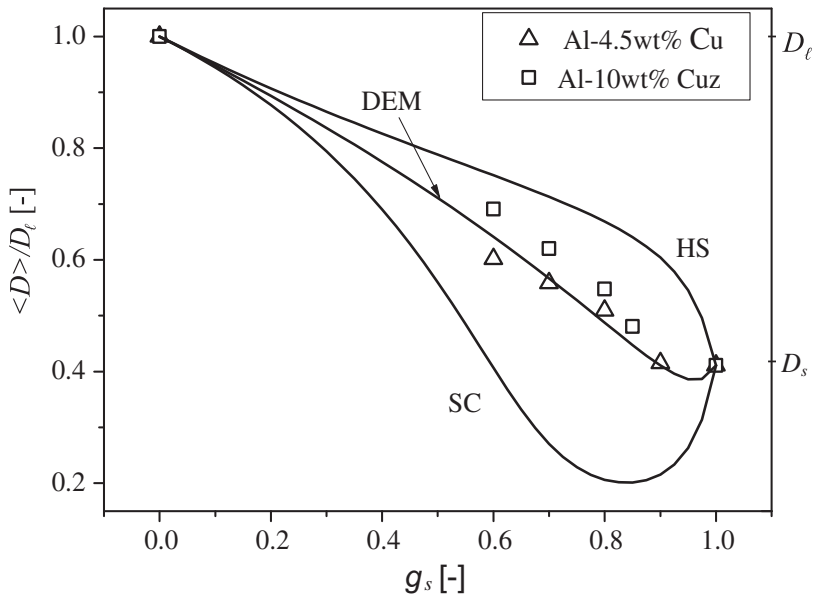

Fig. 4. Simulation results and effective medium approximations for an effective diffusion coefficient $\langle D\rangle$ (normalized by the liquid diffusion coefficient) as a function of the solid fraction.

in order to extract $\langle D\rangle$ (Eq. 18) for both alloys at each solid fraction. The results are summarized in Tables 2 and 3 . The last column of these two tables shows a simple phase fraction-averaging of the diffusion coefficients, i.e. $g_{s} D_{s}+g_{\ell} D_{\ell}$, for comparison.

The effective diffusion coefficients $\langle D\rangle\left(g_{s}\right)$ of these tables are plotted in Fig. 4. They can be compared with the three analytical results from the effective medium theories presented in Section 4.3. As can be seen, the SC approximation,

Table 2

Effective hydrogen diffusion coefficient for an $\mathrm{Al}-4.5 \mathrm{wt} . \% \mathrm{Cu}$ alloy at four different volume fractions of solid.

\begin{tabular}{lllll}
\hline$g_{s}$ & $\langle J\rangle\left(\mathrm{m} \mathrm{s}^{-1}\right)$ & $\nabla\langle X\rangle\left(\mathrm{m}^{-1}\right)$ & $\langle D\rangle\left(\mathrm{m}^{2} \mathrm{~s}^{-1}\right)$ & $g_{s} D_{s}+g_{\ell} D_{\ell}\left(\mathrm{m}^{2} \mathrm{~s}^{-1}\right)$ \\
\hline 0.6 & $8.49 \times 10^{-10}$ & $-1.29 \times 10^{-2}$ & $6.55 \times 10^{-8}$ & $7.05 \times 10^{-8}$ \\
0.7 & $6.39 \times 10^{-10}$ & $-1.05 \times 10^{-2}$ & $6.08 \times 10^{-8}$ & $6.41 \times 10^{-8}$ \\
0.8 & $4.46 \times 10^{-10}$ & $-8.04 \times 10^{-3}$ & $5.55 \times 10^{-8}$ & $5.76 \times 10^{-8}$ \\
0.9 & $2.21 \times 10^{-10}$ & $-4.89 \times 10^{-3}$ & $4.52 \times 10^{-8}$ & $5.12 \times 10^{-8}$ \\
\hline
\end{tabular}

Table 3

Effective hydrogen diffusion coefficient for an $\mathrm{Al}-10 \mathrm{wt} . \% \mathrm{Cu}$ alloy at four different volume fractions of solid.

\begin{tabular}{lllll}
\hline$g_{s}$ & $\langle J\rangle\left(\mathrm{m} \mathrm{s}^{-1}\right)$ & $\nabla\langle X\rangle\left(\mathrm{m}^{-1}\right)$ & $\langle D\rangle\left(\mathrm{m}^{2} \mathrm{~s}^{-1}\right)$ & $g_{s} D_{s}+g_{\ell} D_{\ell}\left(\mathrm{m}^{2} \mathrm{~s}^{-1}\right)$ \\
\hline 0.6 & $8.72 \times 10^{-10}$ & $-1.16 \times 10^{-2}$ & $7.53 \times 10^{-8}$ & $7.05 \times 10^{-8}$ \\
0.7 & $6.07 \times 10^{-10}$ & $-8.98 \times 10^{-3}$ & $6.76 \times 10^{-8}$ & $6.41 \times 10^{-8}$ \\
0.8 & $3.90 \times 10^{-10}$ & $-6.53 \times 10^{-3}$ & $5.97 \times 10^{-8}$ & $5.76 \times 10^{-8}$ \\
0.86 & $2.44 \times 10^{-10}$ & $-4.66 \times 10^{-3}$ & $5.24 \times 10^{-8}$ & $5.38 \times 10^{-8}$ \\
\hline
\end{tabular}


which treats each phase symmetrically, gives very poor results. On the other hand, the Maxwell approximation (the HS upper bound in this case) clearly overestimates the data. The best approximation is obtained with the DEM scheme, which has been reported to be most suitable in cases with large phase contrast, as occurs in the present case, i.e. $\mathfrak{D}_{\ell} / \mathfrak{D}_{s} \approx 40$. This is in line with other work on effective properties where the DEM was found to be a robust predictive scheme even at high phase contrast [22]. Interestingly, $\langle D\rangle$ can be even lower than $D_{s}$ because the remaining liquid droplets at high solid fractions can act as a sink or trap for hydrogen, thus "pumping" hydrogen from the solid and further reducing the average diffusion coefficient.

Note that the considered effective medium theories are valid for isotropic RVEs only. Although RVEs of $560 \times 560 \times 560 \mu \mathrm{m}^{3}$ could be extracted from the tomography experiments, they may not be exactly representative or even isotropic. Moreover, these theories are based on phase fraction information only, assuming a given and simplified microstructure (e.g. spheres in a matrix). They cannot account for the complex and very tortuous morphology of the mushy RVE shown in Fig. 1. As the solid fraction increases towards 1 , there is also a drastic change of the morphology, i.e. the continuous liquid within the solid transforms into isolated liquid droplets.

While the DEM scheme stands, within its limitations, on physically solid ground, it is somewhat cumbersome to work with due to its implicite nature. In the particular case of hydrogen diffusion in $\mathrm{Al}-\mathrm{Cu}$ alloys, the effective diffusion coefficient is quite accurately represented by the simple volume averaged rule of mixture $\langle D\rangle=g_{s} D_{s}+g_{\ell} D_{\ell}$. However, in view of the derivation given above, this particular result must be considered as being fortuitous. It is actually quite simple to run the calculation for the apparently simple case of a two-phase material with identical diffusion coefficients in both phases but a strong partitioning of the diffusing species among the phases. While the rule of mixture would give a constant diffusion coefficient for all phase volume fractions and topologies, the analytical model would yield strongly distinct results. Such a general discussion of diffusion in two- or many-phase media is, however, outside of the scope of this contribution.

\section{Conclusion}

Effective diffusion coefficients as a function of the volume fraction of solid have been calculated on real microstructures obtained via in situ X-ray tomography. For that purpose, a finite volume mesh was produced from the tomography data (solid and liquid voxels becoming solid and liquid nodes, respectively), on which a time-explicit finite volume calculation of hydrogen diffusion was performed. It has been shown that the diffusion equation must be based on the hydrogen chemical potential, or chemical activity, this quantity being continuous across the interface unlike the composition. Several effective medium approximations were compared with the numerical results and the best fit was obtained with the DEM approximation. However, the much simpler volume fraction weighed average $\langle D\rangle=g_{s} D_{s}+g_{\ell} D_{\ell}$ describes the effective diffusion coefficient in these alloys with good accuracy. Such a validated relationship can now be used in integrated models of porosity formation in aluminium alloys.

\section{Acknowledgements}

The tomography part of this work was carried out within the framework of the project ANR-05-BLAN-0286-01 TOMOSOLIDAL, supported by the "Agence Nationale de la Recherche", which is gratefully acknowledged. The authors thank Luc Salvo and Michel Suéry of the SIMAP/GPM2 in Grenoble for help with the X-ray tomography experiments, and Elodie Boller of the ID19 beam line of ESRF Grenoble for technical support. They are also grateful to Samuel Forest from the Ecole des Mines de Paris for providing the furnace.

\section{References}

[1] Hess PD, Turnbull GK. In: Hydrogen in Metals. Champion (PA): ASM; 1974. p. 277.

[2] Anyalebechi PN. In: Light Metals. Warrendale (PA): TMS; 2003. p. 857.

[3] Campbell J. Castings, 3rd ed. Oxford: Butterworth-Heinemann; 2003.

[4] Péquet Ch, Gremaud M, Rappaz M. Metall Mater Trans A 2002;33:2095.

[5] Sabau AS, Viswanathan S. Metall Mater Trans B 2002;33:243.

[6] Couturier G, Rappaz M. Model Simul Mater Sci Eng 2006;14:253.

[7] Lee PD, Hunt JD. Acta Mater 1997;45:4155.

[8] Carlson KD, Lin Z, Beckermann Ch. Metall Mater Trans B 2007;38:541.

[9] Felicelli SD, Wang L, Pita CM, De Obaldia EE. Metall Mater Trans B 2009;40:169.

[10] Atwood RC, Sridhar S, Zhang W, Lee PD. Acta Mater 2000;48:405.

[11] Markworth AJ. J Mater Sci Lett 1993;12:1487.

[12] Rappaz M, Jacot A, Boettinger WJ. Metall Mater Trans A 2003;34:467.

[13] Vernède S, Jarry Ph, Rappaz M. Acta Mater 2006;54:4023.

[14] Limodin N, Salvo L, Boller E, Suéry M, Felberbaum M, Gailliègue S, et al. Acta Mater 2009;57:2300.

[15] Porter DA, Easterling KE. Phase transformations in metals and alloys. 2nd ed. London: Chapman \& Hall; 1992.

[16] Qiu CA, Olson GB, Opalka SM, Anton DL. J Phase Equilib Diffus 2004;25:520.

[17] Torquato S. Random heterogeneous materials. New York: Springer; 2002.

[18] Landauer R. J Appl Phys 1952;23:779.

[19] Maxwell JC. Treatise on electricity and magnetism. Oxford: Clarendon Press; 1873.

[20] Bruggeman D. Ann Phys 1935;24:636.

[21] Hashin Z, Shtrikman S. J Appl Phys 1962;30:3125.

[22] Tavangar R, Molina JM, Weber L. Scr Mater 2007;56:357.

[23] Felberbaum M. PhD thesis, EFPL Lausanne; 2010. 